remaining patients, remission lasted for 9 and 16 months after GCs withdrawal. Each flare required intake of low prednisolone doses for 3-4 weeks.

Conclusion: GCs withdrawal is an achievable goal in SLE and may be attempted after a long-term remission, and possibly after aggressive intensive care in the early stages of SLE.

Disclosure of Interests: None declared

DOI: 10.1136/annrheumdis-2020-eular.3130

\section{AB0390 ASSOCIATION BETWEEN INITIAL SERUM "TUMOR NECROSIS FACTOR-LIKE WEAK INDUCER OF APOPTOSIS" (TWEAK) LEVEL AND TREATMENT RESPONSE IN SYSTEMIC LUPUS ERYTHEMATOSUS (SLE) NEPHROPATHY}

Z. Wailinn ${ }^{1}$, C. Soe ${ }^{2}$, H. M. Win ${ }^{3} .{ }^{1}$ Yangon Specialist Hospital (500 Bedded), Rheumatology Department, Yangon, Myanmar, Burma; ${ }^{1}$ Yangon Specialist Hospital (500 Bedded), Rheumatology Department, Yangon, Myanmar, Burma; ${ }^{3}$ Yangon General Hospital, Medical Department, Yangon, Myanmar, Burma

Background: SLE is a chronic inflammatory immunologic abnormalities disease which produce a number of antinuclear antibodies. The SLE renal involvement is clinically apparent in approximately $50 \%$ patients (Norby et al., 2017). It is very important to introduce the prompt treatment to prevent the permanent end stage renal disease.

Objectives: This study aimed to identify the serum biomarkers that correlate with pretreatment disease activity in patients with SLE nephropathy and predict the treatment outcome so that we may identify the unresponsive cases and switch to the other biologic agents like anti-TWEAK monoclonal antibody in the future.

Methods: This was a hospital-based prospective analytical study conducted from January 2018 to November 2019 in Rheumatology Department, Yangon Specialty Hospital. 88 SLE nephropathy patients with 24-hour urinary protein above $0.5 \mathrm{~g} /$ day planned to have 6 months course of IV cyclophosphamide were enrolled. The paired serum sample of each patient was analyzed by ELISA twice to get the mean serum TWEAK value. Pretreatment SLE disease activity was assessed by the SLEDAI 2k. After the completion of 6 months of aggressive treatment, the treatment response was assessed by measuring the 24 hour urinary protein.

Results: Among the 88 patients, 63 patients $(71.6 \%)$ had completed total 6 -months course and 25 patients ( $28.4 \%$ ) had not completed:11 patients $(12.5 \%)$ expired and 2 patients $(2.27 \%)$ had been changed to other DMARD and 12 patients $(13.63 \%)$ did not attend the follow up clinic. The mean serum TWEAK level was $856 \pm 77 \mathrm{pg} / \mathrm{ml}$ in 88 patients. According to the range of serum TWEAK level, most of the patients had serum TWEAK level of $601-900 \mathrm{pg} / \mathrm{ml}(53.4 \%$ of the study population). There was positive correlation between pre-treatment SLEDAI $2 \mathrm{k}$ score and pretreatment serum TWEAK level $(r=0.464$ and $\mathrm{P}<0.001)$. When the SLEDAI $2 \mathrm{k}$ score was grouped into mild, moderate, high and very high disease activity, the serum TWEAK level also had positive association with the different levels of disease activity $(p<0.001)$. Among 63 treatment completed patients, 55 patients $(87.3 \%)$ were the treatment responders but 8 patients $(12.7 \%)$ were treatment non-responders. There was significant difference in the pretreatment SLEDAI $2 \mathrm{k}$ in terms of disease activity between treatment responder and treatment non-responder $(p<0.001)$. There was significant difference in the pretreatment SLEDAI $2 \mathrm{k}$ in terms of reduction in 24-hours urinary protein between treatment responder and treatment non-responder $(p<0.001)$. There was no significant difference in the level of pretreatment serum TWEAK level between treatment responders and treatment non responders $(p=1.000)$. There was also no significant difference in the pretreatment serum TWEAK level between treatment responders and treatment non-responders in terms of reduction in 24 hours urinary protein $(\mathrm{p}=0.804)$.

Conclusion: Although the pretreatment serum TWEAK level had a positive correlation with pretreatment disease activity of SLEDAI $2 k$, it did not reflect the outcome of the responsiveness to the intensive therapy.

References:

[1] Norby, et al (2017) Outcome in biopsy-proven lupus nephritis: evaluation of

biopsies from the Norwegian kidney biopsy registry. Lupus; 26:88

Acknowledgments: Prof.Chit Soe, Prof.Hlaing Mya Win

Disclosure of Interests: None declared

DOI: 10.1136/annrheumdis-2020-eular.243

\section{AB0391 EFFECT OF HCQ ON LLDAS ACHIEVEMENT IN SLE PATIENTS}

R. Wakiyaa ${ }^{1}$, K. Ueeda ${ }^{1}$, H. Shimada ${ }^{1}$, S. Nakashima ${ }^{1}$, M. Mahmoud Fahmy Mansour ${ }^{1}$, M. Kato ${ }^{1}$, T. Miyagi ${ }^{1}$, Y. Ushio ${ }^{1}$, K. Sugihara ${ }^{1}$, T. Kameda ${ }^{1}$, H. Dobashi'. ${ }^{1}$ Kagawa University, Division of Hematology, Rheumatology and Respiratory Medicine, Department of Internal Medicine, Kagawa, Japan
Background: $\mathrm{HCQ}$ for SLE in Japan has been administered in many cases after approval. Therefore, the effect of additional administration of $\mathrm{HCQ}$ on low disease activity of SLE was considered to be clearer.

Objectives: To clarify the effect of $\mathrm{HCQ}$ treatment on the control of disease activity in SLE patients.

Methods: All SLE patients with low disease activity (LDA) enrolled in this study started additional HCQ treatment from January 2016. All patients with LDA enrolled in this study started HCQ treatment and had been receiving oral $\mathrm{HCQ}$ continuously for at least 3 months without using other immunosuppressive treatments or glucocorticoids. Disease activity was evaluated by SLEDAI, CLASI, and LLDAS, and serum complement values, anti-DNA antibodies, and pro-inflammatory cytokines were analyzed as immunological biomarkers before and after $\mathrm{HCQ}$ treatment.

Results: 52 of 100 patients were enrolled in this study (M:F; 4:48, average age; 40.6 \pm 13.4 ). 24 lupus nephritis patients were in sustained remission. 29 patients $(56 \%)$ achieved LLDAS and 3 patients $(6 \%)$ achieved clinical remission (CR) before $\mathrm{HCQ}$ administration.

Of the 20 patients ( $38 \%$ ) who did not achieve LLDAS before HCQ administration, the LLDAS achievement rates at 3,6 , and 12 months after additional HCQ were $47 \%, 59 \%$, and $81 \%$ (including $12.5 \%$ of $C R$ achievement rates), respectively. Serum levels of MRP8, MRP14, TNF-a, IL-6, VEGF-A, IL-1ra, MIP-1a and IL-2 decreased significantly 3 months after additional $\mathrm{HCQ}$ treatment. In addition, serum levels of MRP8, MRP14, TNF- $a$, IL- 6 and IL-2 also decreased significantly 3 months after additional HCQ treatment despite achieving LLDAS or CR. The expressions of IFN- $a$ didn't decrease significantly in 9 cases that could be detected. The magnitude of the changes in serum MRP8, MRP14, IL- 8 and II-1ra levels in patients with a history of $L N$ was significantly higher than in those without a history of $L N$. The magnitude of the reduction in serum MCP-1 levels in patients not achieving LLDAS with a history of LN was significantly higher than in those without a history of $\operatorname{LN}(\mathrm{p}=0.046)$.

The change of CLASI activity score was correlated with the change in serum levels of MRP14 and MCP-1 with univariate analysis (MRP14: $r=-0.41, p=0.017$ MCP-1: $r=-0.58, p=0.0006$ ). The change of serum C3 levels had a negative correlation with $\mathrm{MCP}-1(\mathrm{r}=-0.33, \mathrm{p}=0.022)$.

The magnitude of the change in serum levels of MRP14, TNF-a, IL-8, MCP1, MIP-1a and IL-1 ra in patients achieving LLDAS were correlated with the change of CLASI activity score with univariate analysis (MRP14: $r=-0.49$, $p=0.041$, TNFa: $r=0.74, p=0.0038$, IL-1 $r a: r=0.66, p=0.038$, MIP-1a: $r=0.63$, $p=0.037$, Figure 1). Moreover, the change of serum C3 and C4 levels in them had a negative correlation with the change of serum MCP-1 levels (Figure 2).
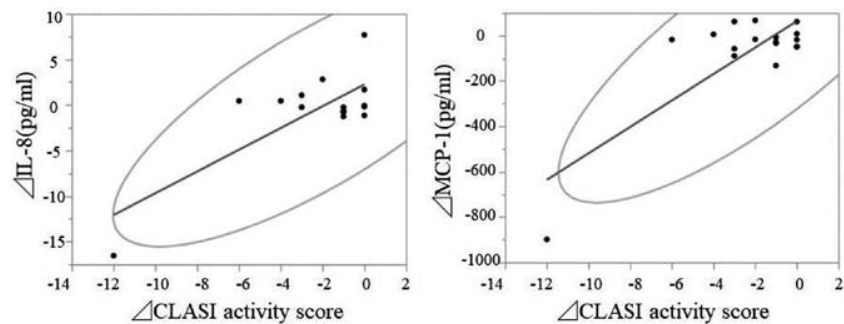

Figure 1. Correlation between change of CLASI activity scores and serum MCP-1 levels in SLE patients with LLDAS (IL-8: $r=0.77, p=0.0007$, MCP-1: $r=0.80, p=0.0001$ ).
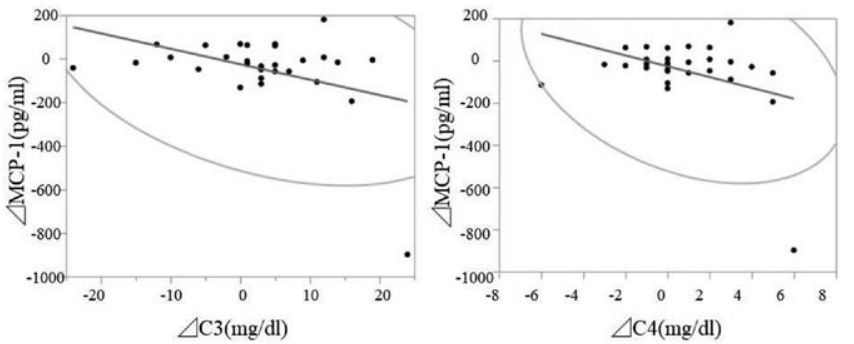

Figure 2. Correlation between change of serum $\mathrm{C} 3$ and $\mathrm{C} 4$ levels and serum MCP-1 levels in SLE patients with LLDAS (C3: $r=-0.40, p=0.028, C 4: r=-0.37, p=0.047$ ).

Conclusion: Additional administration of $\mathrm{HCQ}$ is useful for cytokine control even in LLDAS-achieved cases, and particularly contributes to the improvement of skin lesion.

In addition, regulation of IL-8 and MCP-1 is important for control of renal lesions of SLE, and more control of the activity of SLE

The effect of HCQ on IL-8 and MCP-1 is related to the control of renal lesions in SLE, so that disease activity of more SLE patients might be more controlled disease activity. 
References:

[1] R Wakiya, et al. Hydroxychloroquine modulates elevated expression of S100 proteins in systemic lupus erythematosus. Lupus. 2019;28:826-833

Disclosure of Interests: None declared

DOI: 10.1136/annrheumdis-2020-eular.4149

\section{AB0392 1 THE EFFECT OF HYDROXYCHLOROQUINE ON THE RISK FACTORS FOR ATHEROSCLEROSIS DEVELOPMENT}

R. Wakiya ${ }^{1}$, K. Ueeda ${ }^{1}$, H. Shimada ${ }^{1}$, S. Nakashima ${ }^{1}$, M. Mahmoud Fahmy

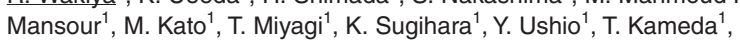
H. Dobashi'. ' Kagawa University, Division of Hematology, Rheumatology and Respiratory Medicine, Department of Internal Medicine, Kagawa, Japan

Background: Systemic lupus erythematosus(SLE) patients, especially patients with lupus nephritis have poor vascular endothelial function and increased cardiovascular(CV) mortality.

Meanwhile, several studies showed hydroxychloroquine(HCQ) has effect on reduction in lipids and thrombosis(1), but the mechanism is unclear.

Objectives: We examined effect of HCQ on adipocytokine expression in SLE patients.

Methods: 52 SLE patients with low disease activity started with HCQ were analyzed before and 3 months after HCQ treatment. 21 SLE patients has past history of lupus nephritis. Serum S100 proteins and adipocytokines were measured by ELISA, and serum inflammatory ctytokine levels were evaluated by Multiplex assay (TNF-a, IL-6, VEGF-A).

Results: Serum adiponectin level was increased significantly 3 months after $\mathrm{HCQ}$ treatment compared with those at baseline (mean change 1.35, Figure 1). SLE patients who achieved LLDAS had a greater increase than those who did not. Additionally, the changes of serum adiponectin levels were associated with those of TNF-a, IL-6, VEGF-A and S100A9 protein, which plays an important role of SLE pathogenesis.

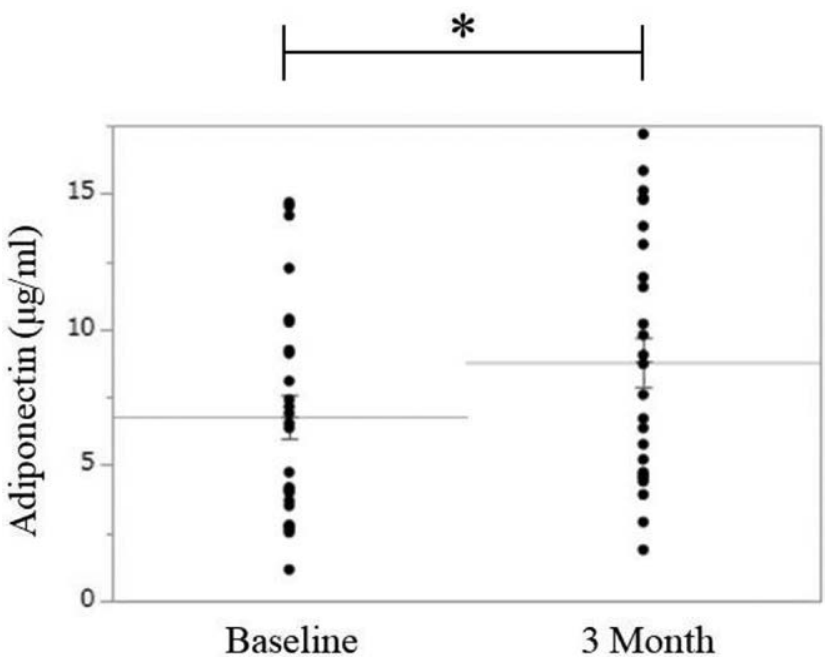

Figure 1. Serum adiponectin levels at baseline were compared with levels after 3 months of $\mathrm{HCQ}$ treatment. Serum adiponectin levels significantly decreasing during $\mathrm{HCQ}$ treatment in SLE patients. For statistical analyses * $p<0.0001, P$ value: Wilcoxon signed-rank test

Conclusion: A HCQ could reduce the risk factors for atherosclerosis along with control of SLE disease activity.

References:

[1] Wallace DJ, et al. Cholesterol-lowering effect of hydroxychloroquine in patients with rheumatic disease: reversal of deleterious effects of steroids on lipids. Am J Med. 1990; 89: 322-6.

Disclosure of Interests: None declared

DOI: 10.1136/annrheumdis-2020-eular.5449

\section{AB0393 \\ EFFICACY OF HYDROXYCHLOROQUINE FOR LUPUS NEPHRITIS IN MAINTENANCE PHASE}

M. Watanabe ${ }^{1}$, Y. Haji ${ }^{1}$, M. Kato ${ }^{1}$, T. Ito ${ }^{1}$, Y. Banno ${ }^{1}$, M. Okada ${ }^{2} .{ }^{1}$ Daido Hospital, Nagoya, Japan; '2St.Luke's International Hospital, Tokyo, Japan

Background: Hydroxychloroquine (HCQ) is an essential drug for systemic lupus erythematosus. But in Japan, chloroquine and HCQ remain unavailable until mid
2015 because of a series of lawsuits about its retinal toxicity in the 1970s. There is insufficient knowledge regarding renal protective effect of $\mathrm{HCQ}$.

Objectives: We aimed to investigate its efficacy of adding HCQ for Lupus nephritis (LN) as a maintenance-phase therapy.

Methods: We conducted an observational retrospective cohort study included patients with $\mathrm{LN}(\mathrm{n}=42)$ in maintenance-phase in Japan. We reviewed medical records of LN patients aged > 18 years who were initiated HCQ from May 2015 to May 2018. Maintenance phase was defined as stabilization in serum creatinine and urinary segment after induction therapy and who achieved complete or partial remission. The annual change of proteinuria was compared between patients on $\mathrm{HCQ}$ who have proteinuria $(>0.5 \mathrm{~g} / \mathrm{gCr}$ ) or not. Other outcome measures were disease flare, dose of steroids, renal and immunologic features.

Results: A total of 42 patients were analyzed and allocated to two groups based on their amount of daily urine protein level: $H C Q$ with proteinuria as group $A(>0.5 \mathrm{~g} / \mathrm{gCr}, \mathrm{n}=14)$ and $\mathrm{HCQ}$ without proteinuria as group $\mathrm{B}(\leqq 0.5 \mathrm{~g} /$ $\mathrm{gCr}, \mathrm{n}=28)$. Both groups were comparable, with mean (SD) age of 36.1 (12.9) years and 37.5 (13.8), female $78.6 \%$ and $92.9 \%$ in each group, mean (SD) disease duration until HCQ of 3.5 (3.25) and 3.3 (2.9) years in group A and group B, with prednisolone dose at base line of 10.3 (7.1) $\mathrm{mg}$ and 7.9 (4.4) $\mathrm{mg}$, respectively. The mean (SD) proteinuria at base line was $1.38(1.11) \mathrm{g} /$ $\mathrm{gCr}$ in group $\mathrm{A}$ and $0.20(0.09) \mathrm{g} / \mathrm{gCr}$ in group $\mathrm{B}$ and after 12 months, proteinuria decreased in group $A(-1.34 \mathrm{~g} / \mathrm{gCr}$ in group $\mathrm{A}$ vs $+0.03 \mathrm{~g} / \mathrm{gCr}$ in group $B$; $\mathrm{p}<.001 ; 95 \% \mathrm{Cl}, 0.305-0.736)$. No relapse was experienced in group $A$ during the study period.

Conclusion: In patients with clinically stable LN but with proteinuria, hydroxychloroquine is a good therapeutic option for achievement of complete remission. Disclosure of Interests: None declared

DOI: 10.1136/annrheumdis-2020-eular.5378

\section{SLE, Sjögren's and APS - clinical aspects (other than treatment)}

AB0394

DOES HIGH DISEASE ACTIVITY IN SYSTEMIC LUPUS ERYTHEMATOSUS PATIENTS INCREASE THE RISK OF CANCER INCIDENCE?

A. Abdalla ${ }^{1}$, F. I. Abdelrahman ${ }^{2} .{ }^{1}$ Aswan University, Rheumatology and Rehabilitation, Aswan, Egypt; ${ }^{2}$ Zagazig University, Rheumatology and Rehabilitation, Zagazig, Egypt

Background: Cancer is known as one of the causes of morbidity and mortality in systemic lupus erythematosus (SLE) patients. It has been thought that SLE activity and stimulation of the immune system predisposes the risk of cancer (1)

Objectives: To investigate the correlation between SLE disease activity and the cancer incidence.

Methods: The study included a cohort of SLE patients, diagnosed according to the American College of Rheumatology classification criteria ${ }^{(2)}$ attending the Rheumatology department, Aswan University in the period from January 2018 to June 2019. We used a questionnaire to screen patients who were diagnosed with cancer. We collected demographic and laboratory data on all screened patients and their disease activity using the Systemic Lupus Erythematosus Disease Activity Index (SLEDAI) ${ }^{(3)}$. For the patients diagnosed with cancer, we recorded age of onset of SLE, age of diagnosis of cancer, type of cancer, treatment received, immunosuppressive regimen (dosage and duration) and cancer outcomes.

Results: The study included 117 patients (95 female, 22 male), mean age $(25.6 \pm 6.5)$ years with mean SLE duration $(7.3 \pm 6.3)$ years and mean SLE $\operatorname{DAl}(9 \pm 8.9) .76 \%$ had lupus nephritis, $62.2 \%$ had hematological abnormalities and $17.8 \%$ had neurophsycatric lupus. $91 \%$ were on corticosteroids (CCS), 33\% on mycophenolate mofetil (MMF), $43 \%$ on azathioprine, $14 \%$ on cyclosporin. $56.8 \%$ were either receiving or had received intravenous cyclophosphamide $(C Y C)$ with a mean cumulative dose $(7.5 \pm 4.7) \mathrm{gm}$. We found 18 (15.3\%) patients (13 female and 5 males) were diagnosed with cancer during the course of SLE with mean age at onset $(31 \pm 3.7)$, mean age at cancer diagnosis $(39.28 \pm 10.77)$, mean SLE duration(18.17 \pm 6.02$)$ and mean SLEDAI $(7.39 \pm 4.19)$. Most of SLE patients with cancer had lupus nephritis ( $89 \%)$ and all cancer patients were on a median dose of CCS 10 (2.5-20) $\mathrm{mg}$ daily for median $10(4-24)$ years. $83.5 \%$ of them had received intravenous CYC prior to the development of cancer with mean total cumulative dose of $(6.7 \pm 4.6) \mathrm{gm}, 67 \%$ received MMF, $33 \%$ received cyclosporine and $50 \%$ received azathioprine. Types of cancer were as follow; $22.2 \% \mathrm{lym}$ phoma, $16.7 \%$ cancer cervix, $16.7 \%$ cancer breast, $11.1 \%$ colorectal cancer, $11.1 \%$ squamous cell carcinoma, $5.6 \%$ leukemia, $5.6 \%$ bronchogenic carcinoma, $5.6 \%$ prostate cancer and $5.6 \%$ cancer thyroid. $66.7 \%$ of them 